\title{
Optimization based Approach for Content Distribution in Hybrid Mobile Social Networks
}

\author{
Rizwan Akhtar ${ }^{1}$, Imran Memon ${ }^{2}$, Zuhaib Ashfaq Khan ${ }^{3}$ and Changda Wang, ${ }^{1, *}$ \\ ${ }^{1}$ School of Computer Science and Communication Engineering \\ Jiangsu University, Zhenjiang P.R China \\ ${ }^{2}$ College of Computer Science \\ Zhejiang University, Hangzhou P.R China \\ ${ }^{3}$ Department of Electrical Engineering \\ Comsats Insititute of Information Technology, Attock, Pakistan
}

\begin{abstract}
This paper presents the new strategy for smooth content distribution in the mobile social network. We proposed a new method, hybrid mobile social network architecture scheme considered as one node of a social community called social super node with higher capacity provides the services of content distribution. We proposed methods and techniques that are introduced to set the criteria to select a social super node (SSN). The simulation results are carried out to measure the correctness of the network performance of the proposed method. These results indicate that the accessing of those content of nearby social super node instead of the content provider is used to improve overall network performance in terms of content end-toend delays, delivery ratio, throughput, and cost.
\end{abstract}

Keywords-Mobile social network; social super node; delays; network performance; end-to-end delays; content delivery

\section{INTRODUCTION}

The mobile social network (MSN) is a future emerging wireless network that provides the combination of social science with wireless communications for purpose of creating mobile networking. The MSN is kind of network which gives different variety of content delivery services and application while involving the social interest of the mobile users. The technique of social networking has been implemented in the research domain of communication technologies to support efficient data exchange, delivery services and sharing [2]. The mobile social networking can be build on the existing mobile network infrastructure i.e. centralized or distributed. The interdependency of such network devices can be exploited by the use of social network analysis concept to provide a better quality of service (QoS).

Different types of network infrastructures utilize in the MSN for the improvement of the performance of content delivery service in MSN such as cellular and Wi-Fi networks, a delay-tolerant network which include opportunistic networks [4]. Other types are MANETs, disconnected delay-tolerant [6], [8], and wireless sensor networks [11]. Nowadays cellular network is most popular and it can be utlized as a backhaul of the network where MSN can be deployed. The cellular networking is able to provide the network infrastructure that can provide social web-based mobile networking services. In this context Facebook, QQ, Twitter, and MySpace are the commonly availabale applications. WI-Fi networks are also supporting the similar services like in cellular networks along with higher content rate but their coverage areas are smaller. In [14] the method of social networking is described to support the teachers to access the desired content through the social network site (SNS) called 'Facebook'. In this method, the teachers can share their content with their community in six different ways by creating online groups. It gives the evidence from a large, open social teachers online group over the time frame of the 12 week period. The findings indicate that large social online open groups in SNSs are useful for the pragmatic advice for teachers.

Content distribution in the MSN is one of the challenging issues that needs to be addressed due to its sparse connectivity along with resource limitation of the mobile devices. To ensure smooth content distribution among the nodes of the MSN, the new appropriate methods and techniques are required for forwarding the data to nodes and also the links for the purpose of increasing the delivery efficiency and reduces the delay. Furthermore, several important factors those are important for content distribution are mobility, time, bandwidth utilization and along with duration of encounters, a freshness of the content, and message duplication available to the users.

Our major contribution of this work is to improve the network performance in term of increasing the probability of successful content delivery and minimizes the delay. It also includes saving the users cost and lowering the system-wide traffic on the backbone of MSN. We proposed a hybrid infrastructure of MSN for efficient content distribution among the users of a certain social community exploiting the social relationship. We select a social super node (SSN) from the social community users of MSN by setting strategies by considering the fluctuating link quality, closeness centrality, mobility pattern and computing resources. Social super node encounters with the content provider server by accessing the cellular network and broadcast to all the users of a certain social community.

This manuscript is organized as follows. Section II explains some related approaches. In Section III we present the analytical model of our scheme. Section IV details our social super node strategies. Section V describes simulation 
results. Section VI concludes the research. Lastly, Section VII Presents the future research direction of the MSN.

\section{RELATED WORK}

The approaches that mainly based on social network analysis such as degree centralities, tie strength, a distance of the links and the node, and mobility pattern is implemented to distribute the data efficiently among the social nodes of the MSN. The affect of the social patterns not only influence the node's interest but their willingness as well (i.e., selfish behavior) for sharing their content [10]. In [1] Peer-to-peer streaming is proposed to enable end-to-end users to communicate and utilize the available resources in the network to share video content. In [3], the time duration of the contact and the frequency of the social pattern (called tie strength) has been used to recognize the contact pattern of the mobile nodes to create a opportunistic contacts social group. In [4], the concept based on flooding is given to distribute the content in the detected social community. However, in such scheme, the flooding message incurs a high volume of the data traffic on the network, which unnecessarily consumes energy, bandwidth usage, and also memory.

In [5] the method of distributed buffer storage in MSN is implemented. Based on such method, a novel solution for the intermittently connected MSN with distributed aid storage for fast and reliable end-to-end content delivery is presented. They proposed an architecture of MSN that is able to offer fast delivery services with the help of distributing buffer storage at the edge of the MSN. It was the attempt to give the solution for connected MSN in the situation, where an offered content rate is higher than the processing content rate of the mobile devices having limited buffer storage. The contribution of that research work is the suppression of information congestion before it occurs by utilizing an extra buffer storage on the access of MSN.

In [12], an algorithm called SMDRA (simple message duplication reduction algorithm) is proposed. This algorithm uses the property of mobility predictability. The limitation of such algorithm is that it incurs larger memory usage, this because it uses graphs pattern to store the desired information related to nodes and edges. In [7], the performance of hierarchical super-node overlays under different configurations is investigated. The configuration parameters include super-node neighbor number (out degree), super node redundancy and request TTL (time to live). In [1], another aspect of the super-node selection for file sharing and streaming application is discussed. The authors developed theoretical methodologies for a super-node selection schemes.

In the work proposed by Nazir et al. [2] discribe that the mobility patterns of the nodes can be predicted if both the record of the time and duration of encounters are maintained. Such information is also beneficial to improve the content delivery ratio. In [8], Costa et al. Present social cast for efficient message routing for publish and subscribe given network by the help of co-location and movement patterns of the nodes mobility pattern together with co-location metric can be utilize to predict the nodes availability so that the appropriate node for forwarding can be selected for content dissemination. In [9], a scheme to direct unconnected social nodes toward connecting mobile social nodes of the social community is proposed. The connected nodes communicate with the main server via a base station, the unconnected nodes can use these connected users to build a relay to obtain connectivity. In [13], an optimized solution based on the content update is proposed. In order to distribute new arrival content as fresh as possible from the server (content provider) to the mobile users.

All the above work are proposed to solve the problems related to content distribution in the MSN. But none of the approach was given for the in shape of the super social node (SSN) across MSN to distribute the content efficiently. Our proposed network architecture is the attempt to fill research gape by taking advantage of the declaring one node as a social super node from the certain social communities.

\section{PROPOSED MSN ARCHITECTURE}

Our proposed scheme is based on a hybrid architecture of MSN that utilizes social super node as an intelligent agent for sharing the content by exploting the social relationship among users. Since our scheme is based on hybrid infrastructure of the MSN that involve both cellular network and ad hoc network. Hybrid MSN guarantee the performance of content delivery and can reduce the network cost.

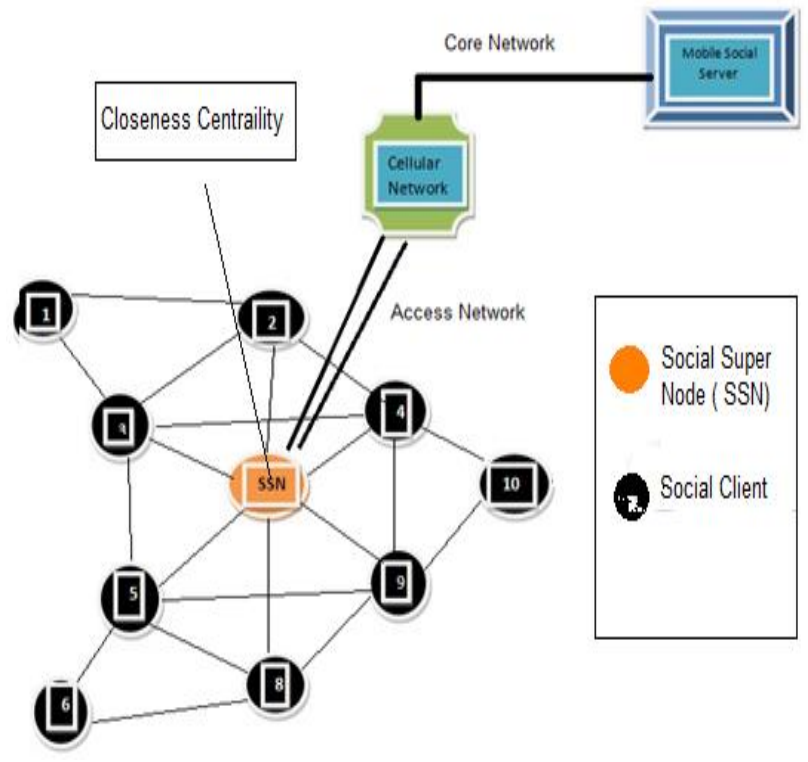

Fig. 1. Social super node of MSN.

In Fig. 1 content provider services declare one node as an SSN on the basis of assumptions in term of a Node, closeness and degree centrality, physical location and storage capacity of the single social community.

In this scheme SSN selection of the $\mathrm{P} 2 \mathrm{P}$ applications is designed to work in backbone networks; SSN is selected to active for a certain period of time to get the content and distribute to the MSN users. Fig. 2 shows there can be multiple communities under base stations of the cellular network. Every community has its own social super node (Table I). 


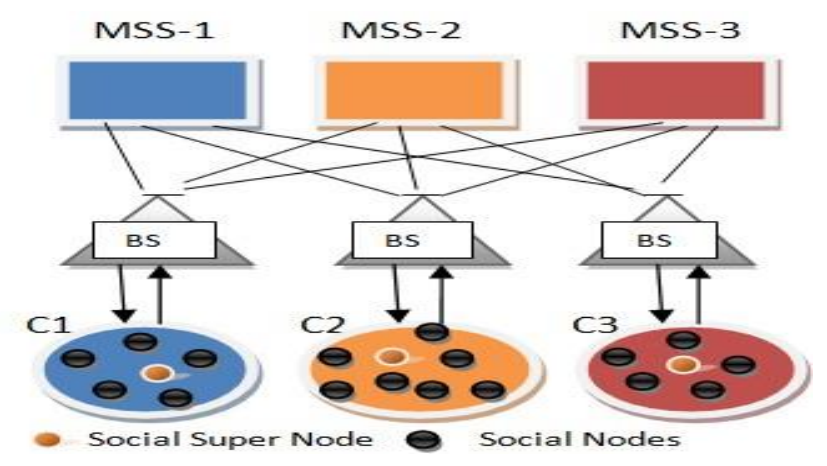

Fig. 2. Multiple social communities.

TABLE I. BINDING TABLE FOR SSN

\begin{tabular}{|l|l|l|}
\hline $\begin{array}{l}\text { Social Super Node } \\
\text { ID }\end{array}$ & IP Addresses & Community ID \\
\hline 2 & 192.168 .2 .0 & $\mathrm{C} 1$ \\
\hline 6 & 192.168 .5 .71 & $\mathrm{C} 2$ \\
\hline 7 & 192.168 .5 .111 & $\mathrm{C} 3$ \\
\hline 3 & 192.168 .4 .42 & $\mathrm{C} 4$ \\
\hline
\end{tabular}

MSS is the mobile social server and $\mathrm{C}$ is a social community. The main contribution of this paper is to define efficient and simple strategies for social super node admission, adapted to wireless ad-hoc network conditions of mobile social network.

\section{SOCIAL SUPER NODE SELECTION}

A social super node can be selected from social community nodes on the assumption of closeness and degree centrality, tie strength, similarity and physical location of the node. The SSN must have higher storage capacity, processing power and energy. The selected SSN of the community can announce it to all associated nodes about its status by broadcasting or MSN server can send a contact message to all the associated node of community and provide information about SSN. Algorithm 1 explains our proposed scheme.

\section{Algorithm 1: Selection / Re-selection of Social Super Node}

Input $\mathrm{N}, \mathrm{C} \quad / / \mathrm{N}$ is number of nodes and $\mathrm{C}$ is the social community Input $\mu, \alpha \quad / / \mu$ is processing rate of $\mathrm{SSN}$ and $\alpha$ is arrival rate Output SSN // Selecting Social Super Node

Begin

$\mathrm{N} \leftarrow \mathrm{C}$

$\mathrm{SSN} \leftarrow \mathrm{N}$

$\mathrm{SSN} \leftarrow \mathrm{C}$

If (TTL $\mathrm{N}=2$-hop count $\& \&$ distance $\mathrm{L}=$ threshold)

// distance of node $\mathrm{N}$ with other nodes

Equation $L_{i}=\sum_{j} q_{i j} L_{i j}$

If $(\mathrm{N} \mu \geq \mathrm{N} \alpha) \quad / /$ processing rate is greater than arrival rate of Node $\mathrm{N}$

Equation $\pi_{j}=C \alpha^{i}=\frac{1-\alpha}{1-\alpha^{j+1}} \alpha^{i} \approx(1-\alpha) \alpha^{i}$

Return $\mathrm{N}=\mathrm{SSN} 1$ // social node $\mathrm{N}$ is set as Social super node 1

If (TTL N $=2$-hop count $\& \&$ distance $L=$ threshold $\& \& N \mu$ $\leq \mathrm{N} \alpha)$
Return N2 = SSN 2 // Set another node N as SSN2 to share the traffic load

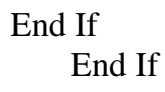

End If

End If

\section{End Begin}

In case of failure of a selected social super node because of overloading, long distance or weak tie strength, MSN server can select another better-positioned node of the community by the assumption of physical location and distance to all the associated nodes and past history of mobility area. MSN services can keep track the social super node and its associated social clients via a cellular system. In case of any social node moves out of the community radio range the MSN server can request them to move back to the community range.

The distance between social super nodes and its node can be calculated as

$$
L_{i=} \sum_{j} q_{i j} L_{i j}
$$

In our system, each super social node of every community $\mathrm{C}$ determines its mean distance to its node. Let Lij denote the number of physical hops between SNi and its j-th node. Then, the mean hop number between the social super node and its node is denoted by $\mathrm{Li}$, can be calculated based on the distance and the hit rate of each node To calculate $\mathrm{Li}$, each Social super node estimates qij for each node $\mathrm{j}$, as follows:

$$
q_{i j}=\frac{\mathrm{NQ}_{\mathrm{ij}}}{\mathrm{NQ}_{\mathrm{i}}}
$$

NQi is Total number of queries received in by SSN and NQij is Number of queries with $\mathrm{j}$ as the MSN server.

In case if the current SSN is far from its community nodes, the admission strategy should seek to replace Selected SSN with a better positioned social super node as SSN-2.

The selected social super node becomes overloaded in a situation where arrival content rate is much higher than the processing rate of super node. The probability of buffer overloaded with its size $\mathrm{J}$ of a SSN can be calculated as

$$
\pi_{i}=C \alpha^{i}
$$

Where $\mathrm{c}$ is the constant value, and $\alpha$ is the exponential controlling the stability margin of the buffer content queue

$$
\lambda(1-\mu) \pi_{j}=\mu(1-\lambda) \pi_{j+1}
$$

$\lambda$ is arrival probability with arrival rate, $\mu$ is departure probability with departure rate and $\pi$ is limiting distribution

$$
\alpha=\frac{(\lambda(1-\mu))}{(\mu(1-\lambda))} \quad \text { and } \quad C=\frac{1-\alpha}{1-\alpha^{j+1}}
$$

Using (3) we get

$$
\alpha^{i} \approx(1-\alpha) \alpha^{i} \pi_{j}=C \alpha^{i}=\frac{1-\alpha}{1-\alpha^{j+1}}
$$


Equation (5) shows that if the value of alpha (Arrival content) is larger, it can be prone to have more contents in the queue and thus SSN can be in the overloaded state.

We assume that the SSN has $100 \mathrm{~KB}$ of the buffer with size $\mathrm{J}$ and arrival content rate (AR) at $\mathrm{SSN}$ via MSN server is at very high than processing rate. We found out the number of times a buffer overflow occurs for a particular time span of 2000 seconds when the same experiment is conducted 100 times at SSN of MSN. The SSN can be overloaded by considering content rate $\lambda=0.43 \mathrm{~KB}$ while processing rate (PR) of contents $\mu=0.40 \mathrm{~KB}$ on the fixed buffer $\mathrm{J}$ of the mobile node of $100 \mathrm{~KB}$. By the help of the probabilistic model, we found out the number of overflows incur for an experiment that is repeated for 100 times across the super social node of MSN. The results show that buffer J overflow occurred 34, 55 and 73 times out of 100 experiments in the conditions when offered content rate is greater, equal and less than the processing rate of the contents. In Fig. 3 red color curve indicates that SSN is in the overloaded state. Whenever an overloaded state is achieved by SSN the admission strategy should seek for the associated social super node as an Assistant Social Super Node (ASSN) for the purpose of sharing loads of the content and can control the arrival traffic rate from the MSN server.

Closeness centrality measures the shortest distance to the entire nodes, whereas degree centrality measures the most direct connections to all nodes of MSN. These two social metrics are also based on the quality of links between nodes, So a selection of SSN also depends on these two social matrices. The best candidate among nodes for the selection of SSN is one who has the shortest distance and most direct connection with all the associated nodes of social community.

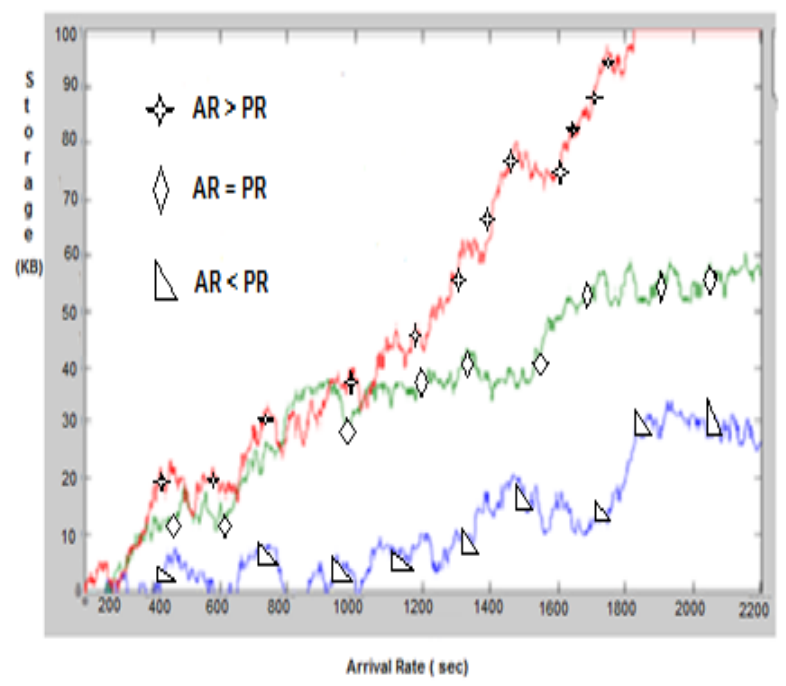

Fig. 3. Curve of overloaded social super node.

\section{PERFORMANCE EVALUATION}

In this section, the simulation results are conducted by using NS-2 to evaluate our proposed scheme for efficient content distribution. In the simulation scenario, we have taken several mobile social nodes and declare one social node from the social community as social super node (SSN) for experiments, a SSN encounter with the mobile social server via cellular network and share the content of all connected friends (nodes) by direct link along with multi-hop connectivity.

Simulation results leverage network performance can be significantly improved if the nodes of the social community can have fresh content from the nearby super node to access instead of content provider at the core of networking. Table II summarizes the parameters used in the simulations.

TABLE II. SYSTEM PARAMETERS FOR SIMULATIONS

\begin{tabular}{|l|l|}
\hline Parameters & Values \\
\hline Number of server & 1 or $2 \ldots . \mathrm{N}$ \\
\hline Number of nodes & $1,30,50,100$ \\
\hline Distance & $10-50 \mathrm{~km} / \mathrm{h}$ \\
\hline Simulation time & $36 \mathrm{hours}$ \\
\hline Bandwidth/good put & $3 \mathrm{Mbit} / \mathrm{s}$ \\
\hline 3G bandwidth/good put & $200 \mathrm{kbit} / \mathrm{s}$ \\
\hline Nodes access network bandwidth & $10 \mathrm{Mbit} / \mathrm{s}$ \\
\hline Content sizes & $1,20,40,60 \mathrm{MB}$ \\
\hline Content popularity & 0.1 to 1.0 \\
\hline \multirow{2}{*}{ Latency (deferred transmission) } & $0,10,50,80,150,300,600$, \\
& $1800,2200 \mathrm{~s}$ \\
\hline
\end{tabular}
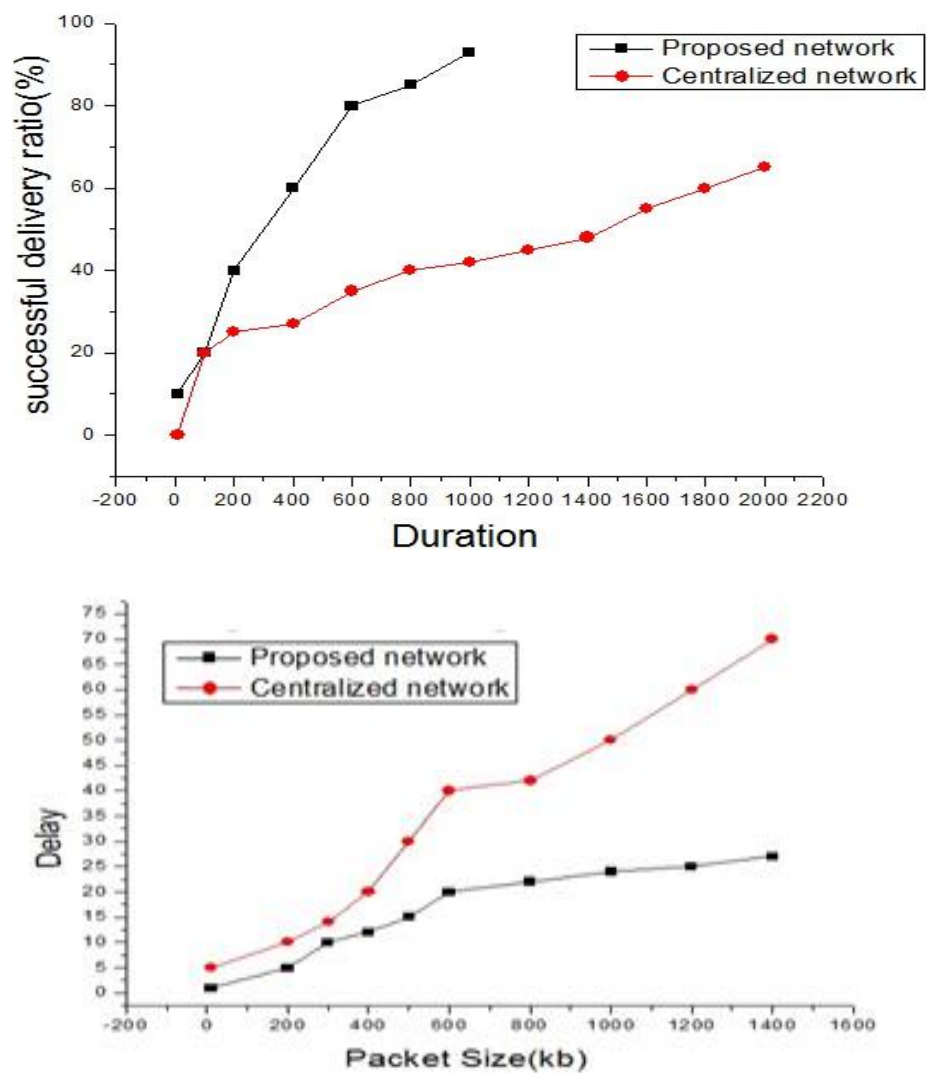

Fig. 4. Successful delivery ratio and delay.

Fig. 4 shows the comparison between the content accessed from source available on access closer to the user with the content access by users from core network by accessing the main social server. Fig. 3 demonstrate that by accessing the content locally from SSN of MSN can increase the successful delivery ratio and reduce the delay. 


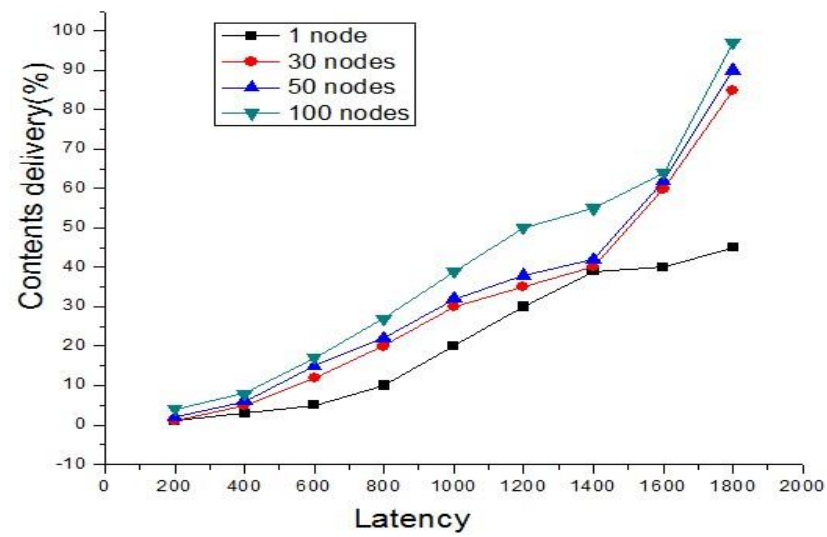

Fig. 5. Latency.

Fig. 5 shows that if nodes access the content of the local SSN would have better latency with a high probability of delivery ratio and lower the delays.

\section{CONCLUSION}

We present the new strategy for smooth content distribution in mobile social network. We proposed a new method, hybrid mobile social network architecture scheme considered as one node of social community called social super node with higher capacity provide the services of content distribution. In this method, techniques are introduced to set the criteria to select a social super node. Our major contribution of this work is to improve the network performance in term of increasing the probability of successful content delivery and minimizes the delay. It also includes saving the users cost and lowering the system wide traffic on the backbone of MSN.

\section{FUTURE WORK}

Although some challenges in the MSN are identified in this research paper, there are still many problems remaining that need to be addressed. Also, there exist many opportunities to improve the effectiveness and efficiency of the MSN. In this section, some of the future possible research recommendations are presented from the perspective of protocol and architecture design in the MSN.

Interoperability is now an important issue because multiple MSN want to communicate at the same with each other. For routing standard protocol, interfaces for exchanging information and signaling of social-based relationship and content delivery among different MSN may be required that can ensure seamless applications. The standard protocols can be useful for data distribution and context awareness and secrecy in the MSNs. Also, the interoperability among mobile users and desktop platforms is an important factor.

Bandwidth allocation within the MSN can be optimized for achieving the best performances However, the impact of mobile social network of exporting social relationship within the radio resources management of several wireless systems has not been yet investigated. In addition, some of the existing optimization formulations ignore the QoS of the MSN applications. Due to the absence of centralized control, radio resources management and also the QoS support are coming more challenging in the distributed architecture of the MSN.

\section{ACKNOWLEDGMENT}

This work was supported by the National Science Foundation of China under grant 61672269 and the Jiangsu Provincial Science and Technology Project under grant BA2015161.

\section{REFERENCES}

[1] Adler M, Kumar R, Ross KW, Rubenstein D, Suel T, Yao DD (2005) Optimal peer selection for P2P downloading and streaming. In: Proceeding of 24th annual joint conference of the IEEE computer and communications societies (INFOCOM), vol 3, pp 1538-1549

[2] F. Nazir, J. Ma, and A. Seneviratne, Time critical content delivery usingpredictable patterns in mobile social networks, in Proc. Int. Conf. Comput. Sci. Eng., Aug. 2009, vol. 4,pp. 1066-1073

[3] R. Cabaniss, S. Madria, G. Rush, A. Trotta, and S. S. Vulli, Dynamic social grouping based routing in a mobile ad-hoc network, [ in Proc. 11th Int. Conf.Mobile Data Manage., May 23-26, 2010, pp. 295-296

[4] E. Yoneki, P. Hui, S. Chan, and J. Crowcroft, A socio-aware overlay for publish/ subscribe communication in delay tolerant networks, in Proc. ACM Symp. Model. Anal. Simul. Wireless Mobile Syst., Oct. 2007, pp. 225-234

[5] Akhtar R, Leng S, Memon I, et al. Architecture of hybrid mobile social networks for efficient content delivery[J]. Wireless Personal Communications, 2015, 80(1): 85-96.

[6] E. M. Daly and M. Haahr, Social network analysis for routing in disconnected delay-tolerant manets, in Proc. ACM Int. Symp. Mobile Ad Hoc Netw. Comput., Sep. 2007, pp. 32-40

[7] Yang B, Garcia-Molina H (2003) Designing a super-peer network. In: Proceeding of 19 th International Conference on Data Engineering (ICDE). Bangalore, India, pp 49-60

[8] P. Costa, C. Mascolo, M. Musolesi, and G. P. Picco, Socially-aware routing for publish-subscribe in delay-tolerant mobile ad hoc networks, IEEE J. Sel. Areas Commun., vol. 26, no. 5, Jun. 2008, pp. 748-760

[9] B. Chelly and N. Malouch, Movement and connectivity algorithms for location-based mobile social networks, in Proc. IEEE Int. Conf. Wireless Mobile Comput. Netw.Commun., Oct. 2008, pp. 190-195

[10] K. Kwong, A. Chaintreau, and R. Guerin, Quantifying content consistency improvements through opportunistic contacts, in Proc. 4th ACM Workshop Challenged Netw., Sep. 25, 2009, DOI: $10.1145 / 1614222.1614230$.

[11] E. Miluzzo, N. D. Lane, K. Fodor, R. Peterson, H. Lu, M. Musolesi, S. B. Eisenman, X. Zheng, and A. T. Campbell, BSensing meets mobile social networks: The design, implementation and evaluation of the CenceMe application, in Proc. ACM Conf. Embedded Netw. Sensor Syst., Apr. 2008, pp.337-350

[12] K. Kawarabayashi, F. Nazir, and H. Prendinger, Message duplication reduction in dense mobile social networks, in Proc. 19th Int. Conf. Comput. Commun. Netw., Aug. 2-5, 2010, DOI: 10.1109/ ICCCN.2010.5560124

[13] S. Ioannidis, A. Chaintreau, and L. Massoulie, Optimal and scalable distribution of content updates over a mobile social network, in Proc. IEEE Int. Conf. Comput. Commun., Apr. 2009, pp. 1422-1430

[14] Kelly, N.; Antonio, A. (2016). "Teacher peer support in social network sites.". Teaching and Teacher Education. $56(1)$ : 2016 138149. doi:10.1016/j.tate.2016.02.007. 